\title{
Téoros
}

Revue de recherche en tourisme

\section{Les récentes études du M.I.C.T. sur le tourisme}

Volume 1, numéro 3, 3e trimestre 1982

URI : https://id.erudit.org/iderudit/1080850ar

DOI : https://doi.org/10.7202/1080850ar

Aller au sommaire du numéro

Éditeur(s)

Université du Québec à Montréal

ISSN

0712-8657 (imprimé)

1923-2705 (numérique)

Découvrir la revue

Citer ce document

(1982). Les récentes études du M.I.C.T. sur le tourisme. Téoros, 1(3), 27-27.

https://doi.org/10.7202/1080850ar d'utilisation que vous pouvez consulter en ligne.

https://apropos.erudit.org/fr/usagers/politique-dutilisation/ 


\section{Les récentes études du M.I.C.T. sur le tourisme}

Lors du Colloque sur la recherche en tourisme, tenu au Centre d'études en tourisme le 3 mars 1982 (voir compte. rendu dans le no 2 de Téoros, p.2), le représentant du ministére de l'Industrie. Commerce et Tourisme n'a pu prendre la parole au panel malgré l'invitation qui luil avait été adressée.

Cette absence a sans doute causé une certaine distorsion dans l'information. mais elle ne peut être imputable aux organisateurs $\mathrm{du}$ colloque. Pour corriger cette distorsion, nous avons ivité le Service analyse et programmation de la Direction générale du tourisme au M.I.C.T. à publier dans cette page les études du ministère traitant du tourisme publiées récemment, réalisées en régie ou à paraître.

La rédaction.

Liste en date d'août 1982

(P) Etude publiée récemment

(R) Document de consultation régie interne

(AP) Document à paraittre prochainement

(PE) Etude publiée par un autre ministêre mais qui est susceptible dinteresser tous ceux qui sont impliqués dans le développement et la promotion du tourisme.

(P) Louise MATTHEWS, Jean Marie LUCAS-GIRARDVILLE, Claire DROUIN, Le tourisme equestre, Service Analyse et Programmation, MICT. Février 1981, 152 pages.

(P) Louise MATTHEWS, Jean-Marie LUCAS-GIRARDVILLE, Un nouveau tourisme: le tourisme actif. I - Le tourisme d'action, Service Analyse et Programmation, MICT, Octobre 1979, 101 pages.

(P) Louise MATTHEWS, Jean-Marie LUCAS-GIRARDVILLE, Un nouveat tourisme: le tourisme actif. II - Le tourisme d'activités, Service Analyse et Programmation, MICT, Août 1981, 92 pages.

(R) Hardy MANHAS, Jean-Marie LUCASGIRARDVILLE, La station touristique Magog-Orford: programme de déve. loppement, Service Analyse et Programmation, MICT, Juin 1981, 220 pages.

(R) Jean-Marie LUCAS-GIRARDVILLE, La station touristique du Mont. Tremblant: programme de développement. Service Analyse et Programmation, MICT, Septembre 1980, 146 pages.

(P) Martine BOIVIN, Louise MATTHEWS, Le tourisme industriel Service Analyse et Programmation MICT, Janvier 1980, 105 pages.
(P) En collaboration sous la responsabilité de Jean-marie LUCAS-GIRARDVILLE, Les croisières au Québec, Service Analyse et Programmation, MICT. Mars 1980,243 pages.

(P) Louise MATTHEWS, Jean-Marie LUCAS-GIRARDVILLE, Le tourisme aै la portee de l'enfant, Service Analyse et Programmation, MICT, Septembre 1980, 105 pages.

(R) Pierre BOIES, La clientèle du ski alpin dans les marchés américains, ontariens et canadiens (Synthèse bibliographiquel, Service Analyse et Programmation, MICT, Décembre 1981, 80 pages.

(PE) Rapport du comité conjoint OPDQ MLCP sur les Villages-VacancesFamilles, La formule VVF au Québec: possibilités et conditions de développement, MLCP. Août 1980,134 pages.

(AP) Patrick CLUZEAU, GUY THIFFAULT, Enquête sur les voyages au Québec et les voyages des Québécois à l'extérieur du Québec en 1981. Service Analyse et Programmation, MICT. 1982.

(PE) Patrick CLUZEAU, Gilles MEUNIER, Dossier technique no 1: l'état de la situation. Une analyse du réseau d'établissements d'accueil et d'hébergement du Québec utilisables pour des vacances familiales, Direction de la planification. MLCP, Juillet 1980, 123 pages.

(PE) Jean DESY, Dossier technique no 2: I'analyse du concept de VVF, MLCP, Juillet 1980, 122 pages.

(PE) Patrick CLUZEAU, Gilles MEUNIER, Dossier technique no 3 : l'étude du marché. Une analyse du comportement des familles québécoises en matière de voyages de vacances en 1979 et de leurs rébactions devant la formule VVF. Direction de la Planifica. tion, MLCP, Octobre 1980, 136 pages.

(P) Sylvie CRÉTE DE NEUVILLE, Patrick CLUZEAU, Sondage 1981 auprès des agences de voyages détaillants du Québec, Service analyse et Programmation, MICT, Mars 1982, 127 pages.

(P) Guy THIFFAULT, Patrick CLUZEAU, Une étude des marchés de Boston, Chicago, Hartford, NewYork, Providence et Washington, D.C. . Service analyse et Programmation, MICT, Avril 1982, 361 pages.

(P) Sylvie CRÊTE DE NEUVILLE, P. CLUZEAU, Sondage 1981 auprès des agences de voyages detaillants des États.Unis et de I'Ontario, Service Analyse et Programmation, MICT, Mai 1982, 134 pages.
(AP) Patrick CLUZEAU, Denis LACROIX Guy THIFFAULT, Enquête sur les voyages des canadiens. Volume 1. Les voyages des Québécois à l'extérieur du Québec en 1978, 1979 et 1980, Service Analyse et Programmation, MICT, 1982

(AP) Patrick CLUZEAU, Denis LACROIX Guy THIFFAULT, Enquête sur les voyages des canadiens. Volume II. Les voyages des Québécois au Québec en 1978, 1979 et 1980, Ser vice Analyse et Programmation. MICT, 1982.

(AP) Patrick CLUZEAU, Denis LACROIX, Guy THIFFAULT, Une enquête sur les voyages des Canadiens. Les voyages des Canadiens des autres provinces au Québec en 1978 et 1980 . Service Analyse et Programmation, MICT, 1982.

(AP) Pierre BOIES, Sondage sur les habi tudes de voyage des Ontriens, sur la perception qu'ils ont du produit touristique québécois et sur leur degré de connaissance de la cam. pagne publicitaire été 1982, Service Analyse et Programmation. MICT, Décembre 1982

(AP) Pierre BolES, Sondage sur le niveau de pénétration de la cam. pagne publicitaire US été 1982. Service Analyse et Programmation. MICT, novembre 1982.

(AP) Gabriel BOUCHARD, Sondage sur les habitudes de voyages des Québécois, sur la perception qu'ils ont du produit touristique québé. cois et sur leur degré de connais. sance de la campagne publicitaire, "C'est beau, c'est grand, c'est à voir", Service Analyse et Program. mation, MICT, date de parution indéterminèe.

(AP) Jean-Marie LUCAS-GIRARDVILLE, Louise MATTHEWS, L'héberge. ment au québec. Evolution et perspective. Une étude sur les modifications de la répartition de l'hébergement, les nouvelles formules d'ave niir, les innovations. Service Analyse et Programmation, MICT, mai 1983

(AP) Jean-Marie LUCAS-GIRARDVILLE, Louise MATTHEWS, Tourisme reli. gieux, Une étude sur les possibilités de cette forme de tourisme. Potentiel. Contraintes. Enquête auprès des ins. titutions. Service Analyse et Programmation, MICT, mai 1982. 